\title{
On the Capacity Region of the Vector Fading Broadcast Channel with no CSIT
}

\author{
Syed Ali Jafar \\ University of California Irvine \\ Irvine, CA 92697-2625 \\ Email: syed@uci.edu
}

\author{
Andrea Goldsmith \\ Stanford University \\ Stanford, CA, 94305 \\ Email: andrea@wsl.stanford.edu
}

\begin{abstract}
We develop an upperbound on the capacity region of an isotropic fading vector broadcast channel in terms of the capacity region of a scalar fading broadcast channel. Using this upperbound we prove the optimality of the Alamouti scheme [1] in a broadcast setting and extend the recent results [2] on the capacity region of the fading scalar non-degraded broadcast channel to fading vector non-degraded broadcast channels. The upperbound is fundamental in that it makes no assumption regarding the distribution of the users' channel magnitudes, the distribution of the additive noise, or the amount of channel information available at the receiver. The scalar upperbound explicitly characterizes the loss of degrees of freedom in a vector broadcast channel when the transmitter has no information about the "direction" of the users' channel vectors.
\end{abstract}

\section{INTRODUCTION}

The capacity region of the fading vector broadcast channel (BC) without channel state information at the transmitter (CSIT) is unknown, except for the special case when all users have identically distributed channels and identically distributed additive noise. In this case all points in the capacity region can be achieved by simply transmitting to only one user at a time and using time-division multiplexing to support multiple users. Thus the system throughput is as if there was only one user in the system. Caire and Shamai's observation [3] that "channel degrees of freedom depend critically upon the availability of channel knowledge at the transmitter" forms the central idea for our work in this paper. In this paper we consider the capacity region of a vector Gaussian BC with $M$ antennas at the transmitter and $K$ users with a single receive antenna at each user. We assume that the transmitter does not have perfect channel state information. However, the transmitter does have perfect knowledge of the ergodic random process governing the successive realizations of the channel fade. We allow memory in the fading process, but we impose one constraint. We consider only isotropic distributions in this paper. By isotropic we mean the following: For the vector $\mathrm{BC}$, since each users' channel can be viewed as an $M$-dimensional vector, it can be described by a "magnitude" and a "direction". Our assumption of isotropic fading means that the transmitter has no knowledge of the "direction" of any user's channel vector. In other words, all directions are equivalent from the transmitter's standpoint. Under fairly general assumptions we show that the capacity region of this vector Gaussian BC is bounded above by the capacity region of a scalar Gaussian BC. The scalar Gaussian BC has only one transmit antenna and the channel gains from the single transmit antenna to each users' receive antenna are given by the Frobenius norm of the original vector channel between the multi-antenna transmitter and that user, scaled by a factor of $\frac{1}{\sqrt{M}}$. The actual upperbound is presented in Theorem 1 . We will refer to this upperbound as the "scalar upperbound". The scalar upperbound is a fundamental result and therefore applies to many system models such as when no CSIR is available, when the transmitter and receiver can only track the users' channel magnitudes [4], with feedback, or when a peak power constraint is assumed. We will start with a very general system model. Additional assumptions for various system models will be specified in later sections.

\section{SySTEM MODEL}

We are interested in the class of channels that can be described by the following system model.

\section{A. Broadcast Channel Model BC-V}

Consider a fading vector broadcast channel with $M$ transmit antennas at the base station and $K$ users with a single receive antenna at each user given by the input/output relationship

$$
\begin{aligned}
Y_{t}^{[1]} & =\mathbf{H}_{t}^{[1]} \mathbf{X}_{\mathbf{t}}+Z_{t}^{[1]} \\
& \vdots \\
Y_{t}^{[K]} & =\mathbf{H}_{t}^{[K]} \mathbf{X}_{\mathbf{t}}+Z_{t}^{[K]}
\end{aligned}
$$

where for user $k$ at time instant $t, \mathbf{H}_{t}^{[k]}$ is the $1 \times M$ channel vector, $Y_{t}^{[k]}$ is the received scalar signal and $Z_{t}^{[k]}$ is additive noise. $\mathbf{X}_{t}$ is the $M \times 1$ complex vector symbol transmitted by the base station at time instant $t$.

Let the average transmit power be $P$, so that

$$
\mathrm{E}\left[\operatorname{Tr}\left(\mathbf{X} \mathbf{X}^{\dagger}\right)\right] \leq P .
$$

Figure 1 shows the channel model for BC-V with two users. The channel fade and noise processes are ergodic and stationary. We allow the channel fade process to have memory. Thus, successive realizations of the channel and/or the noise may be correlated. For simplicity, the time index is suppressed. The precise definition of isotropic fading channels is as follows: 


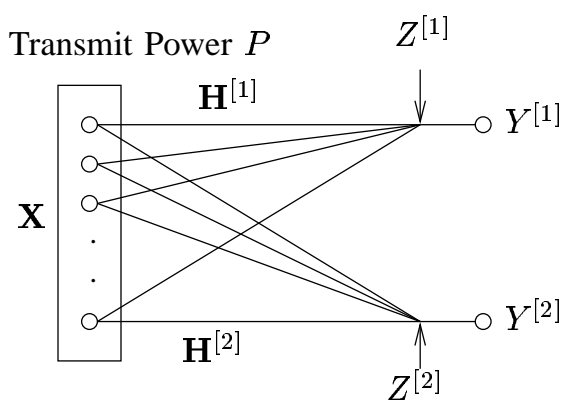

Fig. 1. BC-V

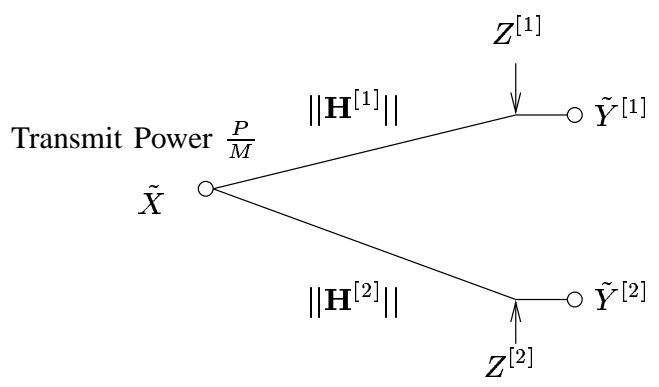

Fig. 2. BC-S
Isotropic Fading: We consider the class of channels that can be described as

$$
\mathbf{H}^{[k]}=\boldsymbol{\Phi}^{[k]} h^{[k]}
$$

where $\boldsymbol{\Phi}^{[k]}$ is a $1 \times M$ isotropically random complex unit vector and

$$
h^{[k]}=\left\|\mathbf{H}^{[k]}\right\|,
$$

the norm of the instantaneous channel vector is a non-negative scalar random variable independent of $\boldsymbol{\Phi}^{[k]}$. It is important to note that each users' channel norm and noise may have a completely different distribution.

Recall that an isotropically random vector is one whose distribution is not affected by multiplication with a unitary matrix. It is the mathematical way to capture the notion that the vector is equally likely to point in any direction in the $M$ dimensional vector space. An example of a channel that belongs to this class is the Rayleigh fading channel with additive white Gaussian noise (AWGN) where each users' channel vector $\mathbf{H}^{[k]}$ consists of i.i.d. complex Gaussian elements $\mathbf{H}^{[k]} \sim \mathcal{N}\left(0, \alpha_{k}^{2}\right), 1 \leq i \leq M$. Isotropic fading is a fairly general and realistic assumption, capturing all fading models where the transmitter does not have enough information to discriminate between various transmit directions. The generality of this model is captured in the fact that it applies to any distribution for the channel norm $h^{[k]}$. Also, it can be argued that over sufficiently long intervals any wireless channel may be modeled as isotropically random in the absence of any side information.

Since we do not assume any specific distribution for the channel norm $h^{[k]}$ or the additive noise $Z^{[k]}$, the BC-V fading vector broadcast channel model is not degraded (stochastically or physically), more-capable or less-noisy in general.

We relate the capacity region of the class of vector broadcast channels represented by $\mathbf{B C}-\mathbf{V}$ to the capacity region of the class of scalar broadcast channels presented next.

\section{B. Broadcast Channel Model BC-S}

Associated with the broadcast channel BC-V, we define another channel model, BC-S, with input/output relationship

$$
\begin{aligned}
\tilde{Y}^{[1]} & =h^{[1]} \tilde{X}+Z^{[1]} \\
& \vdots \\
\tilde{Y}^{[K]} & =h^{[K]} \tilde{X}+Z^{[K]}
\end{aligned}
$$

Notice that the input $\tilde{X}$ is a scalar, and each users' channel is also a scalar equal to the norm of the corresponding original vector channel. The new transmit power constraint is

$$
\mathrm{E}\left[|\tilde{X}|^{2}\right] \leq \frac{P}{M} .
$$

Figure 2 shows the channel model for BC-S with two users.

\section{ThE Vector BC WITH No CSIT AND PERfECT CSIR}

The first system model we consider is where the transmitter has no channel state information while the receiver has perfect channel state information. The following theorem presents the scalar upperbound in this context.

Theorem 1: [The Scalar Upperbound]: The capacity region of the vector fading broadcast channel BC-V with no CSIT and perfect CSIR is contained within the capacity region of the scalar fading broadcast channel BC-S with no CSIT and perfect CSIR.

Thus the capacity region of BC-S upperbounds the capacity region of BC-V. Note that Theorem 1 does not assume that the channels are degraded in any fashion. Also, the theorem does not assume any specific distribution for the additive noise or the channel magnitudes.

Proof of Theorem 1: We start with the following lemma:

Lemma 1: The capacity region of the broadcast channel BC-V is unaffected by the assumption

$$
\boldsymbol{\Phi}^{[1]}=\boldsymbol{\Phi}^{[2]}=\cdots=\boldsymbol{\Phi}^{[K]}=\boldsymbol{\Phi}
$$

where $\boldsymbol{\Phi}$ is an isotropically distributed complex random vector. Cover proved in [5] that the capacity region of a broadcast channel depends only on the marginal distributions. Since the assumption (7) does not affect the marginal distributions $p\left(Y^{[1]} \mid \mathbf{X}\right), p\left(Y^{[2]} \mid \mathbf{X}\right), \cdots, p\left(Y^{[K]} \mid \mathbf{X}\right)$ of the users' channels, 
the capacity region is unaffected by this assumption. Henceforth, in this section we assume that (7) is true, i.e. the users' channel vectors are parallel.

We need the following definitions:

$$
\begin{aligned}
& X^{\|} \triangleq<\mathbf{X}, \boldsymbol{\Phi}> \\
& X^{\perp} \triangleq\left\|\mathbf{X}-X^{\|} \Phi\right\|
\end{aligned}
$$

Thus, $X^{\|}$is a complex scalar random variable representing the projection of the transmitted vector symbol $\mathbf{X}$ along the direction of the instantaneous channel $\Phi . X^{\perp}$ is a non-negative real scalar random variable representing the magnitude of the projection of the transmitted vector symbol $\mathbf{X}$ along the null space of the channel. Note that the projections do not depend on the particular user because the assumption (7) implies all instantaneous channel realizations are parallel. The power in the transmitted symbol $\mathbf{X}$ is related to $X^{\|}$and $X^{\perp}$ as

$$
\operatorname{Tr}\left[\mathbf{X X}^{\dagger}\right]=\|\mathbf{X}\|^{2}=\left|X^{\|}\right|^{2}+X^{\perp 2}
$$

Lemma 2:

$$
\mathrm{E}_{\boldsymbol{\Phi}}\left[\left|X^{\|}\right|^{2}\right]=\frac{1}{M} \operatorname{Tr}\left(\mathbf{X X}^{\dagger}\right)
$$

In other words, regardless of the input distribution, the average transmitted power along the channel is always a fraction $1 / M$ of the overall transmit power. The proof is quite straightforward. Since the transmitter has no channel knowledge, the transmitted vector symbol $\mathbf{X}$ is independent of the instantaneous channel direction $\boldsymbol{\Phi}$. Consider an $M \times M$ isotropically distributed random unitary matrix $\mathbf{U}$ independent of $\mathbf{X}$. Without loss of generality we can assume that successive realizations of $\boldsymbol{\Phi}$ are the successive realizations of the first column of $\mathbf{U}$. Each column of $\mathbf{U}$ is an isotropically distributed random unit vector. Since each column is identically distributed the expected power in the transmitted signal projected along each column is identical. However, together all columns span the entire space of possible transmit signals. Thus the total transmit power $P$ is split equally among the directions corresponding to each column of the random matrix $\mathbf{U}$. This proves the result of Lemma 2 .

With the definitions (8) and (9), we can represent the broadcast channel BC-V input/output relationship as

$$
\begin{aligned}
Y^{[1]} & =h^{[1]} X^{\|}+Z^{[1]} \\
Y^{[2]} & =h^{[2]} X^{\|}+Z^{[2]} \\
& \vdots \\
Y^{[K]} & =h^{[K]} X^{\|}+Z^{[K]}
\end{aligned}
$$

Notice the similarity between BC-S and (12), and the implied power constraint from Lemma 2 and (2)

$$
\mathrm{E}_{\mathbf{H}, \mathbf{X}}\left[\left|X^{\|}\right|^{2}\right]=\frac{P}{M} \text {. }
$$

Note that for BC-V since the transmitter does not know $\boldsymbol{\Phi}$ it cannot choose $X^{\|}$, i.e, the transmitter can only choose $\mathbf{X}$ and the channel realization determines $X^{\|}$. The transmitter does not know the channel in BC-S either, but because BC-S is a scalar broadcast channel there is no uncertainty about the direction of the channel vector. Thus the difference between $\mathbf{B C}-\mathbf{V}$ and $\mathbf{B C}-\mathbf{S}$ is that in $\mathbf{B C}-\mathbf{S}$ we allow the transmitter to code directly over $X^{\|}$. Theorem 1 states that this can only enhance the capacity region. In light of the observations pointed out above, the result of Theorem 1 may already seem intuitive at this point. A formal argument is presented next.

In order to prove Theorem 1 we will show that any vector codebook designed for BC-V can also be used on the scalar broadcast channel $\mathbf{B C}-\mathbf{S}$ with the same resulting performance. Thus, any rate vector achievable in $\mathbf{B C}-\mathbf{V}$ is also achievable in BC-S.

Let the users' messages be denoted by the respective indices $W_{k}$ and the users' rates be denoted by $R_{k}$ so that $1 \leq W_{k} \leq 2^{N R_{k}}$ where $N$ is the length of the codeword measured in number of channel uses. Consider any codebook $\mathbf{X}\left(W_{1}, W_{2}, \cdots, W_{K}\right)$ for the original fading vector broadcast channel BC-V. The codebook maps each message tuple to an $M \times N$ codeword matrix $\mathbf{X}\left(W_{1}, W_{2}, \cdots, W_{K}\right)$ such that the $t^{t h}$ column of the matrix represents the $M$-dimensional complex vector symbol that is transmitted on the $M$ base station transmit antennas at time instant $t$. We denote the $t^{t h}$ column of the codeword matrix $\mathbf{X}\left(W_{1}, W_{2}, \cdots, W_{K}\right)$ as $\left[\mathbf{X}\left(W_{1}, W_{2}, \cdots, W_{K}\right)\right]_{\bullet t}$.

We wish to use the same codebook on the fading scalar broadcast channel BC-S where the base station has only one transmit antenna. So we need to map the columns of $\mathbf{X}\left(W_{1}, W_{2}, \cdots, W_{K}\right)$ onto scalar inputs of BC-S, $\tilde{X}$. This is done as follows. We assume that along with the codebooks, the transmitter and the receiver are provided with identical copies of a pre-generated sample path of i.i.d. realizations of isotropically distributed unit vector $\boldsymbol{\Phi}$ before the beginning of communication. So the transmitter and receiver use the same sequence of i.i.d. isotropically distributed unit vectors $\boldsymbol{\Phi}_{t}$. At the $t^{t h}$ channel use, the transmitter of BC-S uses this $\boldsymbol{\Phi}_{t}$ and computes the projection of the corresponding transmit symbol $\left[\mathbf{X}\left(W_{1}, W_{2}, \cdots, W_{K}\right)\right]_{\bullet t}$ onto $\mathbf{\Phi}_{t}$ to obtain the scalar complex transmit symbol $\tilde{X}_{t}$. That is,

$$
\tilde{X}_{t}=<\left[\mathbf{X}\left(W_{1}, W_{2}, \cdots, W_{K}\right)\right]_{\bullet t}, \mathbf{\Phi}_{t}>.
$$

This is where we need Lemma 2, as it guarantees that the power constraint for BC-S is satisfied.

$$
\mathrm{E}\left[\left|\tilde{X}_{t}\right|^{2}\right] \leq \frac{P}{M}
$$

For the $t^{t h}$ channel use on BC-S, plugging (14) into (5) gives us the input/output relationship,

$$
\begin{aligned}
\tilde{Y}_{t}^{[1]} & =h_{t}^{[1]} \mathbf{\Phi}_{t}\left[\mathbf{X}\left(W_{1}, W_{2}, \cdots, W_{K}\right)\right]_{\bullet t}+Z^{[1]} \\
\tilde{Y}_{t}^{[2]} & =h_{t}^{[2]} \mathbf{\Phi}_{t}\left[\mathbf{X}\left(W_{1}, W_{2}, \cdots, W_{K}\right)\right]_{\bullet t}+Z^{[2]} \\
& \vdots \\
\tilde{Y}_{t}^{[K]} & =h_{t}^{[K]} \mathbf{\Phi}_{t}\left[\mathbf{X}\left(W_{1}, W_{2}, \cdots, W_{K}\right)\right]_{\bullet t}+Z^{[K]}
\end{aligned}
$$

But this is identical to the input/output for BC-V when the same codebook is used and when the actual channel for user $k$ at time $t$ is $\mathbf{H}_{t}^{[k]}=h_{t}^{[k]} \boldsymbol{\Phi}_{t}$, and the channel is known to the 
receiver. Since the codebook is designed for the same channel distribution, it must perform at least as well on BC-S as it does on BC-V. In other words, the probability of error for this codebook is the same whether it is used on BC-V or BC$\mathbf{S}$. Thus, any rate vector achievable on $\mathbf{B C}-\mathbf{V}$ is also achievable on BC-S. This completes the proof of Theorem 1.

Next, while staying with the assumption of no CSIT and perfect CSIR, we present a few interesting cases where the upperbound of Theorem 1 is achievable, i.e. the capacity regions of BC-V and BC-S are identical.

\section{Optimality of Alamouti Scheme}

In this section we consider a specific sub-class of fading broadcast channels within the class represented by BC-V. In particular, we impose the following additional assumptions on the vector broadcast channel BC-V:

1) $M=2$, i.e. the base station has two transmit antennas.

2) We assume that the additive noise is Gaussian and the noise process is memoryless.

3) Coherence time $T \geq 2$.

The following theorem presents our main result in this section:

Theorem 2: The capacity region of the vector fading broadcast channel BC-V with no CSIT, perfect CSIR and assumptions 1, 2, and 3 listed above is identical to the capacity region of the scalar fading broadcast channel BC-S with no CSIT, perfect CSIR and assumptions 2 and 3. Also, in this case, the Alamouti scheme can be used to achieve capacity on the vector broadcast channel using scalar codes.

The full proof of Theorem 2 is provided in [4], where we show that applying the Alamouti space-time coding scheme [1] transforms the channel from BC-V to BC-S .

Interestingly, we do not even need the assumption (7). So, even if all users' channels are independent, applying Alamouti space time coding transforms the vector fading $\mathbf{B C}-\mathbf{V}$ into the scalar fading BC-S with half the transmit power. Thus, clearly for this sub-class of fading broadcast channels, the upperbound of Theorem 1 is tight, and the two capacity regions are identical. This is true without any assumptions on the distribution of the channel magnitudes, i.e. the channel may be non-degraded in general.

\section{TWO USER FADING GAUSSIAN CHANNEL}

Our main aim in this section is to extend the recent scalar Gaussian BC results of Shamai et. al. [2] to vector Gaussian BC. Starting with the system models BC-V and BC-S we make the following additional assumptions:

1) $K=2$, i.e., there are two users.

2) We assume AWGN.

Let $\mathcal{C}^{[1]}(p)$ and $\mathcal{C}^{[2]}(p)$ denote the single user capacities of users 1 and 2 respectively, when the total transmit power is $p$. So the single user capacities for BC-V are $\mathcal{C}^{[1]}(P)$ and $\mathcal{C}^{[2]}(P)$ respectively. Define the following two conditions:

Condition 1: $d(\alpha)=\mathcal{C}^{[1]}(\alpha P)-\mathcal{C}^{[2]}(\alpha P)$ is a non-negative function for all $\alpha \in[0,1]$.

Condition 2: $d(\alpha)<d(1)$ for all $\alpha \in[0,1)$.
For the scalar fading BC under these conditions, the capacity is known [2]. Note that these conditions do not make the fading broadcast channel degraded. The following theorem states our main result in this section:

Theorem 3: Whenever condition 1 or condition 2 is satisfied, the capacity region of the vector fading broadcast channel BC-V with two users, additive white Gaussian noise, no CSIT and perfect CSIR is identical to the capacity region of the scalar fading broadcast channel BC-S with two users, AWGN, no CSIT and perfect CSIR.

We prove Theorem 3 in [4] by showing that with Gaussian codebooks the vector broadcast channel BC-V achieves the entire capacity region of the corresponding scalar broadcast channel BC-S. Since the latter is an upperbound on the capacity region of $\mathbf{B C}-\mathbf{V}$, the scalar upperbound is shown to be tight and the two capacity regions are identical.

\section{The Vector BC with No CSIT And No CSIR}

In this section we consider the vector fading broadcast model BC-V when channel state information is available to neither the transmitter nor any of the receivers. For a single user Rayleigh fading channel with additive White Gaussian noise at the receiver the case of no CSIT and no CSIR has been explored by Marzetta and Hochwald [6]. Their work shows that the degrees of freedom corresponding to multiple transmit antennas depend dramatically upon the channel coherence time and the channel state information at the receiver. Specifically, they show that with no CSIR or CSIT, increasing the number of transmit antennas beyond the coherence time $T_{c}$ does not increase capacity. The following theorems state our main results for this case:

Theorem 4: The capacity region of the vector fading broadcast channel BC-V with no CSIT and no CSIR is contained within the capacity region of the scalar fading broadcast channel BC-S with no CSIT and no CSIR.

The proof is nearly identical to the proof of Theorem 1 . The details are in [4]. Also, in [4] we show that the scalar upperbound is loose in the following case.

Theorem 5: The scalar upperbound is not tight for a single user channel with $M>1$ transmit antennas, i.i.d. Rayleigh fading, no CSIT, no CSIR and coherence time $T_{c}=1$.

This example is treated in detail in [4]. The scalar upperbound only captures the loss of degrees of freedom due to lack of CSIT. Lack of CSIR is a greater limitation and as shown by [6] it also affects the transmitter in that some transmit antennas may be redundant. By eliminating the redundant transmit antennas before applying the scalar upperbound, a better upperbound is readily obtained [7]. In [7] the results of [6] for Rayleigh fading point-to-point vector channel are extended to the more general case of isotropic fading nondegraded broadcast channels. It is not known if the improved upperbound obtained in this manner is tight.

\section{LOSS OF DEGREES OF FREEDOM}

In this section we return to our starting point: the observation that "channel degrees of freedom depend critically upon 
the availability of channel knowledge" [3]. While [3] makes this observation in the context of a Gaussian broadcast channel with no CSIT, perfect CSIR, and i.i.d. users, it is a fundamental property of vector channels. Marzetta and Hochwald's results on the capacity of Rayleigh fading multiple antenna channel with no CSIT or CSIR can be interpreted in the same light. For a fast fading channel (channel coherence time $T_{c}=1$ ), the transmitter and receiver do not have sufficient information about the channel and therefore capacity does not increase with the number of transmit antennas. In other words, the multiple spatial dimensions offered by the vector input channel are lost because the amount of channel knowledge is insufficient. The scalar upperbound is a result along the same lines. It shows how the degrees of freedom corresponding to multiple antennas are limited by the transmitter's inability to identify and distinguish between the directions of different users' channels. In this section we consider the asymptotic limit of a large number of transmit antennas, i.e. $M \rightarrow \infty$. The additional assumptions on the $\mathbf{B C}-\mathbf{V}$ model for this section are:

1) Noise is AWGN.

2) Large number of transmit antennas: $M \rightarrow \infty$.

For any user $k \in\{1,2, \cdots, K\}$, first note that with isotropic fading the power in each component of the vector channel $\mathbf{H}^{[\mathbf{k}]}$ is the same. So let us define

$$
\mathrm{E}\left[\left|H_{1}^{[k]}\right|^{2}\right]=\cdots=\mathrm{E}\left[\left|H_{M}^{[k]}\right|^{2}\right] \triangleq \Gamma^{[k]}, k \in\{1,2, \cdots, K\} \text {. }
$$

The following Theorem presents our main result of this section:

Theorem 6: In the limit as $M \rightarrow \infty$, the capacity region of the vector fading broadcast channel $\mathbf{B C}-\mathbf{V}$ with no CSIT and AWGN is identical to the capacity region of the corresponding scalar fading broadcast channel BC-S. The asymptotic sum rate capacity $C_{\Sigma}=\max _{k \in\{1,2, \cdots, K\}} \log \left(1+P \Gamma^{[k]}\right)$.

The proof is provided in [4].

Theorem 6 states that as the number of antennas gets large, the total throughput is identical to that of a single user system. This is true regardless of the distribution of the users' magnitudes, whether the channel is degraded or non-degraded, and whether the CSIR or channel magnitude feedback is available. The loss of degrees of freedom is evident as the sum rate capacity does not grow with the number of users or the number of transmit antennas.

It has been pointed out by Shamai and Tuninetti [2] that for scalar fading Gaussian broadcast channels it is not known if the sum rate capacity is always achieved by transmitting only to the user with the largest single user capacity. Through Theorem 6 we have established that this is indeed true in the limit of many transmit antennas. We have also shown that if the transmitter does not have any information about the channel direction, then at best the thoughput can not be more than the largest single user capacity without fading. Neither more users nor more transmit antennas can improve the throughput beyond that.

\section{CONCLUSION}

We present an upperbound on the capacity region of a vector broadcast channel with $M$ transmit antennas and a single receive antenna at each user. The bound is in terms of the capacity region of a scalar broadcast channel where the transmit power is reduced by a factor $\frac{1}{M}$ and the scalar channel gains are the magnitudes of the corresponding vector channels. We call this bound the scalar upperbound.

The scalar upperbound is a fundamental result that applies to a wide range of scenarios. It applies whenever, conditioned on the side information available at the transmitter the channel distribution is isotropic: i.e., the transmitter has no information about the relative spatial direction of each user's channel vector. Thus, the scalar upperbound is applicable irrespective of the noise distribution, each user's channel fade distribution, channel or noise memory, and the side information available at the receiver. In addition to the system models considered in this paper, in [4] we use the scalar upperbound under various additional assumptions such as: perfect channel output feedback, peak power constraint, magnitude feedback, and multiple receive antennas. In particular, we obtain the capacity region of a vector fading Gaussian BC with perfect CSIR and magnitude feedback: i.e., when each user's instantaneous channel norm is made available to the transmitter.

The scalar upperbound characterizes the loss of degrees of freedom in a vector broadcast channel when the transmitter has no information about the "direction" of the users' channels. It shows how the degrees of freedom corresponding to multiple users and multiple transmit antennas are lost by the transmitter's inability to identify and distinguish between the directions of different users' channels. It underscores the need for directional information at the BC transmitter.

The scalar upperbound is also a valuable tool for proving capacity results for isotropically fading vector broadcast channels. By reducing the vector broadcast channel to a scalar channel we are able to make use of the known capacity results for scalar channels. Although the scalar upperbound may not be tight in general, in many cases it is tight and this allows us to determine the capacity region of the vector broadcast channel.

\section{REFERENCES}

[1] S. M. Alamouti, "A simple transmitter diversity scheme for wireless communications," IEEE J. Select. Areas Commun., vol. 16, pp. 14511458, Oct. 1998.

[2] D. Tuninetti and S. Shamai, "On two user fading Gaussian broadcast channels with perfect channel state information at the receivers," in Proceedings of IEEE Int. Symp. Inform. Theory, 2003.

[3] G. Caire and S. Shamai, "On the achievable throughput of a multiantenna Gaussian broadcast channel," IEEE Trans. Inform. Theory, vol. 49, pp. 1691-1706, July 2003.

[4] S. Jafar and A. Goldsmith, "Vector broadcast channels with no CSIT: Capacity region bounds and loss in degrees of freedom," In preparation.

[5] T. M. Cover, "Broadcast channels," IEEE Trans. Inform. Theory, vol. 18, pp. 2-14, Jan. 1972.

[6] T. Marzetta and B. Hochwald, "Unitary space-time modulation for multiple-antenna communications in Rayleigh flat fading," IEEE Trans. Inform. Theory, vol. 46, pp. 543-564, March 2000.

[7] S. Jafar, "Too much mobility limits the capacity of ad-hoc networks," In preparation. Preprint available upon request. 